\title{
Laser resonators formed by two nanoparticles
}

\author{
Xiaohua $\mathrm{Wu}^{a}$, Wei Fang ${ }^{a}$, Alexey Yamilov ${ }^{a, b}$, Andrey A. Chabanov ${ }^{a, c}, \mathrm{Hui} \mathrm{Cao}^{a}$ \\ ${ }^{a}$ Dept. of Phys. and Astro., Northwestern Univ., 2145 Sheridan Rd, Evanston, IL, 60208 \\ ${ }^{b}$ Dept. of Phys., Univ. of Missouri-Rolla, 1870 Miner Circle, Rolla, MO, 65409 \\ ${ }^{c}$ Dept. of Phys. and Astro., Univ. of Texas-San Antonio, San Antonio, TX, 78249
}

\begin{abstract}
We demonstrate lasing in a cavity formed by two Mie scatterers in a dye colloidal solution. Like a FabryPerot cavity, the feedback mechanism for lasing is based on back scattering from each particle. Strong light amplification in between the scatterer pair not only compensate its large diffraction loss, but also help to choose the particular pair out of many scatterers in the suspension to form the laser cavity. Such cavity selection is facilitated by a careful designed cone shaped excitation geometry. Detailed experimental studies on the threshold behavior, spectral characteristic of lasing emission, and output directionality are presented. A simple theoretical model provides qualitative explanation for this lasing phenomenon.
\end{abstract}

Keywords: Mie scattering, dye solution, two-scatterer laser

\section{INTRODUCTION}

In a regular laser cavity such as a Fabry-Perot or ring cavity, a scatterer is detrimental to lasing as it increases loss by scattering photons out of the cavity. However, in a gain medium containing many scatterers, multiple scattering increases the path length or dwell time of light in the gain medium, thus enhances light amplification. The laser whose feedback mechanism is based on optical scattering instead of reflection has been a subject of extensive studies ${ }^{1}$ after the pioneering work of Letokhov and coworkers. ${ }^{2,3}$ Since multiple scattering is usually caused by random fluctuation of refractive index, such laser is called a random laser. In addition to provide feedback for lasing, scattering can also lead to spatial confinement of laser emission in micron-sized volume. ${ }^{4}$ A micro random laser of size $\sim 1 \mu \mathrm{m}$ has been demonstrated. ${ }^{5}$ However it contains more than ten thousand scatterers (nano-particles). In 1998 Wilhelmi proposed a laser resonator composed of a pair of dipole scatterers with gain medium in between. ${ }^{6}$ The feedback is provided by back scattering from each scatterer and the large diffraction loss is compensated by strong gain in between. In this paper, we report the first experimental realization of the two-scatterer lasers in colloidal dye solutions. Detailed experimental studies on the threshold behavior, spectral characteristic of laser emission and output directionality are presented.

Though the feedback is caused by scattering from particles, the two-scatterer laser is very different from random lasers. It can be treated as a Fabry-Perot cavity with end mirrors replaced by two nano particles. While this two-scatterer laser may seem simple, its realization in a colloidal solution is not trivial. Even in a dilute suspension of particles, there are many pairs of particles. Careful design of excitation geometry has to be used to facilitate certain scatterer pair over the others. We will show that the two particles which form the lasing cavity have the largest possible separation inside the gain volume for maximum one-path light amplification. This means, the two-scatterer cavity with the lowest lasing threshold is not the one that has the highest quality factor, but the one that ensures the largest light amplification. Such gain facilitated lasing modes have been reported in $2 \mathrm{D}$ random system recently. ${ }^{7}$ The other scatterers in the solution are detrimental to lasing from this two-scatterer cavity, and it is replaced by random lasing at high scatterer concentration. A simple theory model is proposed to explain experimental results from the two-scatterer laser as well as its transition to random laser at higher scatterer concentration.

Further author information: h-cao@northwestern.edu, Tel: 1 847-467-5452 Fax: 847-491-9982

Laser Beam Control and Applications, edited by Alexis V. Kudryashov, Alan H. Paxton, Vladimir S. Ilchenko, Adolf Giesen, Detlef Nickel, Steven J. Davis, Michael C. Heaven, J. Thomas Schriempf, Proc. of SPIE Vol. 6101, 61010M, (2006) - 0277-786X/06/\$15 · doi: 10.1117/12.647859

Proc. of SPIE Vol. 6101 61010M-1 


\section{EXPERIMENTS}

We performed experiments on several systems with passive scattering particles embedded in active homogeneous media. The scatterers are $\mathrm{TiO}_{2}$ particles of radius $200 \mathrm{~nm}, \mathrm{ZnO}$ particles of radius $38 \mathrm{~nm}, \mathrm{SiO}_{2}$ particles of radius $220 \mathrm{~nm}$. The nano-particles are suspended in a laser dye solution, e.g., Rhodamine 640 perchlorate or Rhodamine 590 chloride in diethylene glycol (DEG) or methanol. The experimental results obtained with different scatterers are similar, despite of the differences in particle size and refractive index. In the following, we will use the $\mathrm{TiO}_{2}$ particles as an example to demonstrate the lasing phenomena.

A small amount of $\mathrm{TiO}_{2}$ (rutile) particles, with an average radius of $200 \mathrm{~nm}$, were dissolved in the DEG solution of rhodamine 640 perchlorate dye. To prevent flocculation, the $\mathrm{TiO}_{2}$ particles were coated with a thin layer of $\mathrm{Al}_{2} \mathrm{O}_{3}$. DEG was chosen as the solvent instead of widely-used methanol because of the facts that (i) the windows of the quartz cuvette that contains the methanol solution were coated with a layer of $\mathrm{TiO}_{2}$ particles, whereas such coating was not observed for the DEG solution; (ii) the viscosity of DEG is about 30 times larger than methanol, thus the sedimentation of $\mathrm{TiO}_{2}$ particles in DEG is much slower. In our experiment, the particle density $\rho$ ranged from $1.87 \times 10^{8} \mathrm{~cm}^{3}$ to $5.6 \times 10^{10} \mathrm{~cm}^{3}$. The scattering mean free path was estimated by $l_{s}=1 / \rho \sigma_{s}$, where $\sigma_{s}$ is the scattering cross section of a $\mathrm{TiO}_{2}$ spherical particle with radius $200 \mathrm{~nm}$. The value of $l_{s}$ varied from $1.07 \mathrm{~cm}$ to $35 \mu \mathrm{m}$. The dye molarity $M$ also changed from 3 to $10 \mathrm{mM} / \mathrm{L}$. Right before the lasing experiment, the suspension was placed in an ultrasonic bath for 30 minutes to prevent sedimentation of the particles. During the experiment, the solution was contained in a quartz cuvette that was $1.0 \mathrm{~cm}$ long, 1.0 $\mathrm{cm}$ wide and $4.5 \mathrm{~cm}$ high. The dye molecules in the solution were optically pumped by the frequency-doubled output $\left(\lambda_{p}=532 \mathrm{~nm}\right)$ of a mode-locked Nd:YAG laser (25ps pulse width, $10 \mathrm{~Hz}$ repetition rate). The pump beam was focused by a lens into the solution through the front window of the cuvette. The radius of the pump spot at the entrance to the solution was about $20 \mu \mathrm{m}$. The emission from the solution was collected in the backward direction of the incident pump beam. A second lens focused the emission into a fiber bundle (FB), which was connected to the entrance slit of a spectrometer with cooled CCD array detector. The spectral resolution was $0.06 \mathrm{~nm}$.

We started the experiment with a solution of $M=5 \mathrm{mM} / \mathrm{L}$ and $\rho=3.0 \times 10^{9} \mathrm{~cm}^{-3}$. At low pumping level, the emission spectrum featured the broad spontaneous emission band of rhodamine 640 molecules. Above a threshold pump intensity, discrete narrow peaks emerged in the emission spectrum, and their intensities grew rapidly with increasing pumping. This behavior corresponded to the onset of lasing. The lasing peaks could be as narrow as $0.12 \mathrm{~nm}$. Their frequencies changed from pulse to pulse (shot). We repeated the experiment with solutions of different particle density but the same dye concentration. Lasing was observed only within certain range of particle density. Figure 1 show the spectra of emission from five solutions taken at the same incident pump pulse energy $0.4 \mu \mathrm{J}$. Each spectrum was integrated over 25 shots. Only a relatively broad amplified spontaneous emission (ASE) peak was observed for the neat dye solution, whereas a few discrete lasing peaks emerged on top of the ASE spectrum at small particle concentration $\rho=1.87 \times 10^{8} \mathrm{~cm}^{-3}$. Increasing particle density to $1.87 \times 10^{9} \mathrm{~cm}^{-3}$ led to an increase in the number of lasing peaks and the peak intensity. However, when $\rho$ increased further to $1.3 \times 10^{10} \mathrm{~cm}^{-3}$, the lasing emission started to decrease. Eventually at $\rho=5.0 \times 10^{10}$ $\mathrm{cm}^{3}$ lasing peaks disappeared. A further increase of the incident pump pulse energy to $1.2 \mu \mathrm{J}$ resulted in an ASE peak at longer wavelength, shown in the right inset of Fig. 1. The red shift of the ASE peak might be caused by reabsorption of emission which was enhanced by strong scattering at high particle density. In left inset of Fig. 1, the incident pump pulse energy at the lasing threshold $E_{t}$ is plotted against the particle density $\rho$. At the lasing threshold, the slope of emission intensity versus pump energy exhibited a sudden jump (inset of Fig. 2). At $\rho=1.87 \times 10^{8} \mathrm{~cm}^{-3}$, lasing started at $0.2 \mu \mathrm{J}$. As $\rho$ increased to $1.87 \times 10^{9} \mathrm{~cm}^{-3}, E_{t}$ decreased gradually to $0.15 \mu \mathrm{J}$. Then it remained nearly constant with a further increase of $\rho$. The threshold started to rise at $\rho=1.3 \times 10^{10} \mathrm{~cm}^{-3}$, then went up quickly with $\rho$. At $\rho=5.0 \times 10^{10} \mathrm{~cm}^{3}$, no lasing peaks were observed up to the maximum pump pulse energy $2.0 \mu \mathrm{J}$ we used, although at $1.0 \mu \mathrm{J}$ an ASE peak appeared at longer wavelength.

The threshold behavior illustrates that lasing in the dilute suspension of particles is fundamentally different from random lasing that is based on multiple light scattering. In the latter, the lasing threshold decreases with 


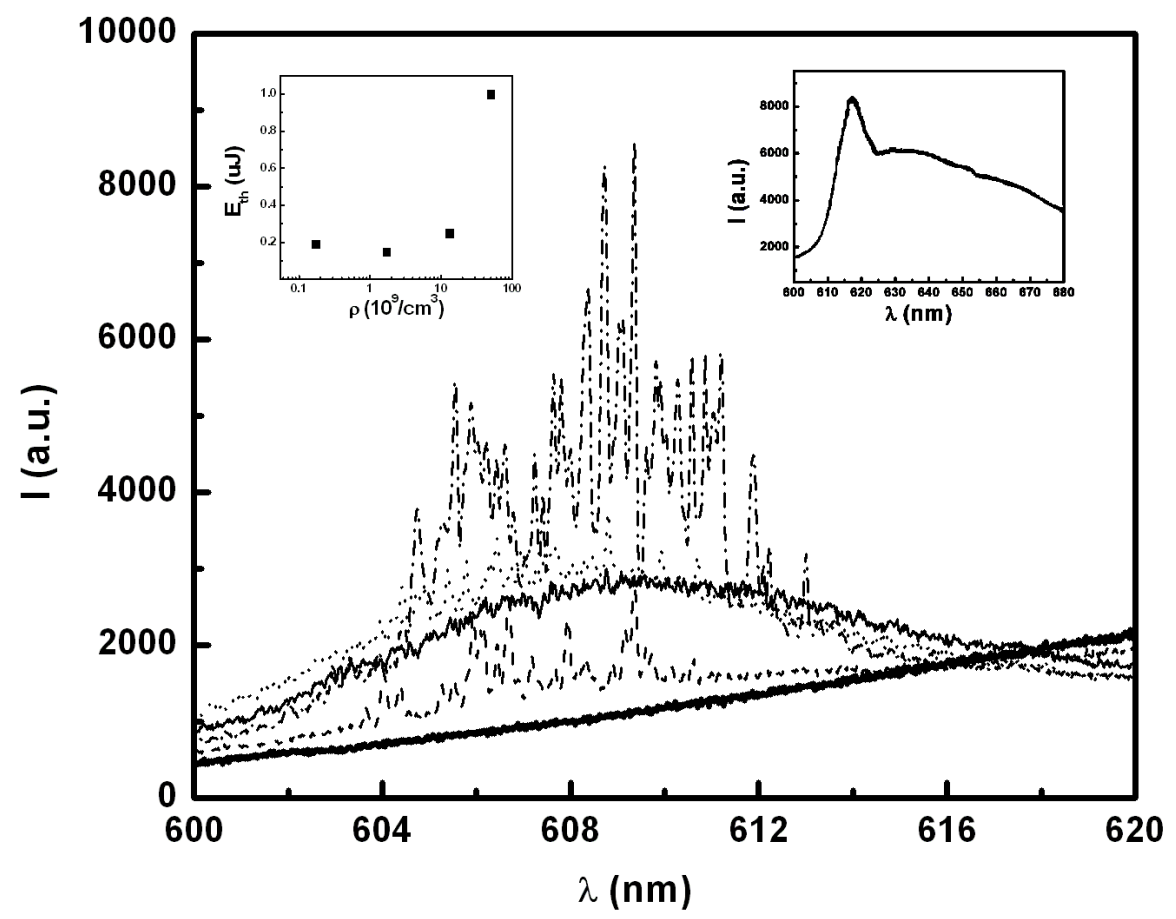

Figure 1. Emission spectra of $5 \mathrm{mM} / \mathrm{L}$ Rhodamine 640 dye in DEG with different particle concentration. 1: Neat dye solution (thin solid), 2: $1.87 \times 10^{8} / \mathrm{cm}^{3}($ dot $), 3: 1.87 \times 10^{9} / \mathrm{cm}^{3}$ (dash dot), $4: 1.3 \times 10^{10} / \mathrm{cm}^{3}($ dash $), 5: 5.0 \times 10^{10} / \mathrm{cm}^{3}($ thick solid). All spectra were taken at $0.4 \mu \mathrm{J}$ and integrated for 25 shots. Inset left is threshold pump pulse energy for solutions with different $\rho$. Inset right is the ASE spectrum of solution with $5.0 \times 10^{10} / \mathrm{cm}^{3} \mathrm{TiO}_{2}$ excited at $1.2 \mu J$.

an increase in the amount of scattering. ${ }^{8}$ However, the particles must play an essential role in the new lasing process which does not occur in the neat dye solution. One possibility is lasing within individual particles that serve as laser resonators. However, this explanation contradicts the experimental observation from our emission angular measurement, which showed the lasing emission is highly directional. The left inset of Fig. 2 is a sketch of our directionality measurement setup. A fiber bundle was placed at the focal plane of the lens. It was scanned with fine steps parallel to the focal plane. At each step, the spectrum of emission into a particular direction was recorded. The emission angle $\theta$ was computed from the fiber bundle position. Its range was limited by the diameter of the lens to about 14 degree. $\theta=0$ corresponds to the backward direction of the incident pump beam. In each spectrum, the emission intensity was integrated over the wavelength range 604-612 nm in which the lasing peaks were located. Figure 2 is a plot of the integrated emission intensity versus the output angle $\theta$ at $\rho=3.0 \times 10^{9} \mathrm{~cm}^{-3}$. Although the spontaneous emission at low pumping was isotropic, the lasing emission was strongly confined to the backward direction of the incident pump beam. Its full width at half maximum (FWHM) was about 4 degree, which was close to the divergence angle of the focused pump beam. To check the effect of reflection by the front window of cuvette on lasing, we rotated the cuvette horizontally and repeated the measurement. As shown in the right inset of Fig. 2, $\phi$ is the angle between the incident pump beam and the normal of the front window. Similar lasing phenomena were observed except a slight increase of the lasing threshold. The lasing emission was always confined to the backward direction of pump beam even when $\phi$ was much larger than the divergence angle of the focused pump beam. This result demonstrated that the front window of the cuvette did not play an essential role in the lasing process. Figure 2 also shows the angular distribution of ASE from a solution of higher particle density $\left(\rho=5 \times 10^{10} \mathrm{~cm}^{-3}\right)$. The integrated intensity of ASE (at longer wavelength) was nearly constant over the angular range of detection. 


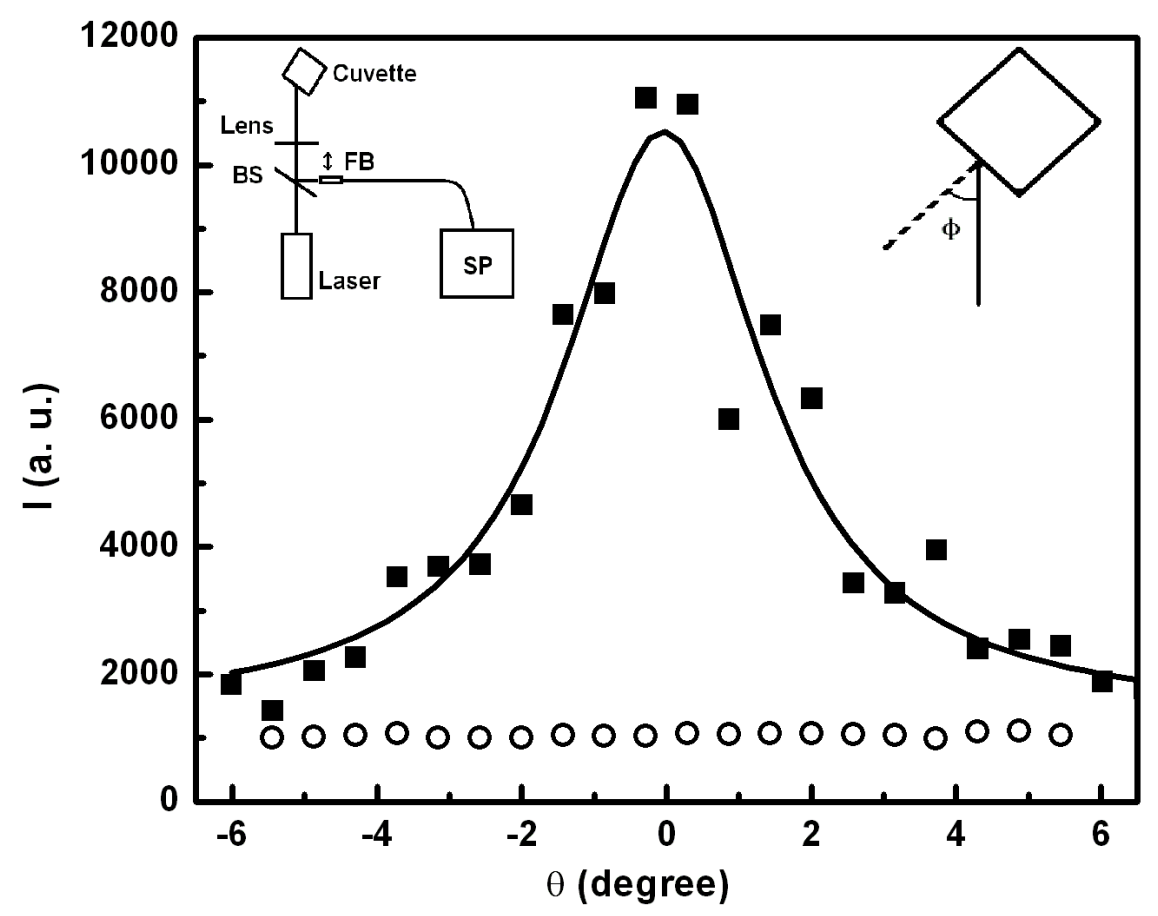

Figure 2. The angular distribution of lasing emission from $5 \mathrm{mM} / \mathrm{L}$ Rhodamine 640 in $\mathrm{DEG}$ with $3.0 \times 10^{9} / \mathrm{cm}^{3} \mathrm{TiO}{ }_{2}$. Each data point is the integration of emission spectrum from $604 \mathrm{~nm}$ to $612 \mathrm{~nm}$ at each angle. Zero degree is the backward direction of pumping. Inset left is a sketch of experimental setup, BS: beam splitter, SP: spectrometer, FB: fiber bundle. Inset right indicates the tilted cuvette from pumping direction.

To understand the directionality of lasing emission from the dilute suspension, the pumped region was imaged through a side window of the cuvette. The measurement setup was sketched in the inset of Fig. 3(a). Emission from the excited region was collected through the side window by a $5 \mathrm{x}$ objective lens and imaged onto a CCD camera. Spectrum was taken simultaneously by partitioning the signal with a beam splitter (BS). Figure 3(a) compares the spectrum of emission through the side window to that through the front window of the cuvette from the same solution $\left(\rho=3 \times 10^{9} \mathrm{~cm}^{-3}, M=5 \mathrm{mM} / \mathrm{L}\right)$ under identical pumping condition. The spectrum of emission from the front window exhibited large lasing peaks. However, only spontaneous emission was obtained through the side window, and it shifted to longer wavelength as a result of reabsorption in the unpumped solution between the excitation region and side window. To calibrate the reabsorption, we measured the spontaneous emission spectra at low pumping from both front and side windows. The magnitude of reabsorption was estimated from the intensity ratio of emission through the side window to that through the front. Based on this estimation, we concluded that the absence of lasing peaks in the side emission spectrum at high pumping was not caused by reabsorption. This confirmed the result of directionality measurement presented in the last paragraph. More importantly, the image of spontaneous emission intensity distribution taken through the side window revealed the shape of the excited region in the solution. As shown in Fig. 3(b), the excitation volume at low particle density had a cone shape. The length of the cone was much larger than its base diameter. Unfortunately, we could not get the exact length of the cone from the image, because near its end the spontaneous emission was too weak to be recorded by the CCD camera. At high particle density, the shape of excitation volume changed to hemisphere as shown in Fig. 3(c). This change was caused by increased scattering 
of pump light. In Fig. 3(b) $\rho=3.0 \times 10^{9} \mathrm{~cm}^{-3}$, the scattering mean free path $l_{s}$ at the pump wavelength $\lambda_{p}=532 \mathrm{~nm}$ was estimated to be $800 \mu \mathrm{m}$. The (linear) absorption length $l_{a}$, obtained from the transmission measurement of neat dye solution, was about $50 \mu \mathrm{m}$ at $\lambda_{p}=532 \mathrm{~nm}$. Strong pumping in the lasing experiment could saturate the absorption of dye molecules, leading to an increase of $l_{a}$. Nevertheless, $l_{a}$ was still shorter than $l_{s}$, and scattering of pump light was much weaker than absorption. Thus the shape of the excitation volume was nearly identical to that in the neat dye solution. In Fig. $3(\mathrm{c}) \rho=5.0 \times 10^{10} \mathrm{~cm}^{-3}, l_{s}$ was shortened to $53 \mu \mathrm{m}$. Scattering of pump light became much stronger. As a result, the cone was replaced by a hemisphere. The image of excitation volume provided some clue to high directionality of lasing emission at small $\rho$ and non-directionality of ASE at large $\rho$ in Fig. 2. At low particle density, stimulated emission in the cone-shaped gain volume was the strongest along the cone due to the longest path length. Since the cone was parallel to the incident pump direction, lasing was confined to this direction. At large $\rho$, emitted photons experienced multiple scattering while being amplified in the hemisphere-shaped gain volume. Hence, the ASE was nearly isotropic.
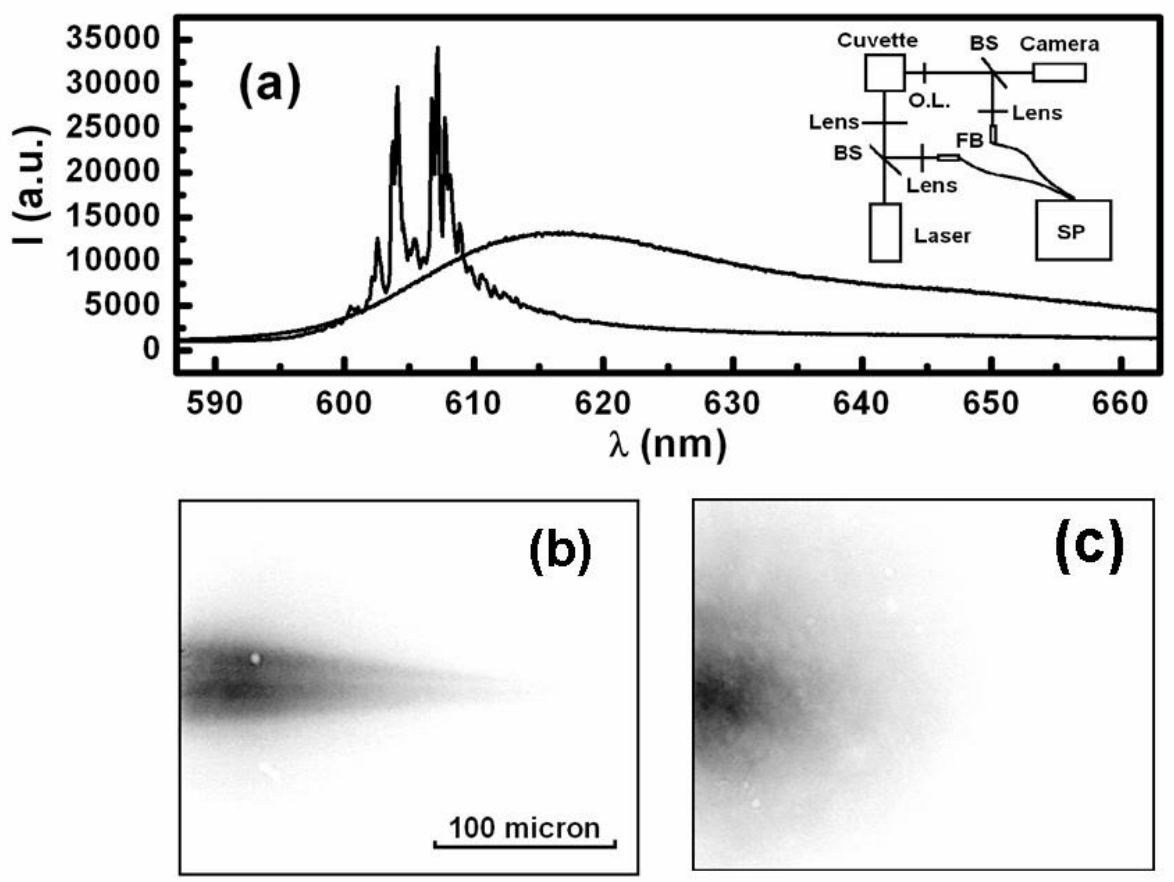

Figure 3. (a): Emission spectrum taken through side (no sharp peaks) and front window (with sharp peaks) of cuvette. Inset is the setup sketch for side collection and image experimental setup, BS: beam splitter, SP: spectrometer, FB: fiber bundle. (b) and (c): Side image of excitation region of $5 \mathrm{mM} / \mathrm{L}$ Rhodamine 640 in DEG with two $\mathrm{TiO}_{2}$ concentration: $3.0 \times 10^{9} / \mathrm{cm}^{3}(\mathrm{~b})$ and $5.0 \times 10^{10} / \mathrm{cm}^{3}(\mathrm{c})$.

Even though the directionality of lasing emission could be explained qualitatively by the geometry of gain volume, it was still not clear how lasing cavities were formed in the dilute suspension where light transport was ballistic. We examined the lasing spectra more carefully by taking single-shot emission spectra with the same setup for Fig. 1. Surprisingly, most single-shot spectra featured almost equally spaced lasing peaks. Figure 4(b) is an example of single-shot emission spectrum taken from the solution of $\rho=3.0 \times 10^{9} \mathrm{~cm}^{-3}$ and $M=5 \mathrm{mM} / \mathrm{L}$. The spectral correlation function $C_{I}(\Delta \lambda) \equiv\langle I(\lambda) I(\lambda+\Delta \lambda)\rangle /\left\langle I(\lambda)^{2}\right\rangle$ was computed for the spectrum in Fig. 4(a) (middle) and plotted in Fig. 4(b) (middle). The regularly spaced correlation peaks revealed the periodicity of lasing peaks. Despite that the lasing peaks completely changed from shot to shot, the peak spacing was nearly the same. From the wavelength spacing $\Delta \lambda$ of the lasing peaks, we derived the cavity length $L_{c}$ with the formula 
of a Fabry-Perot cavity, namely $L_{c}=\lambda^{2} / 2 n_{e} \Delta \lambda \sim 200-300 \mu \mathrm{m}$, where $n_{e}$ is the effective index of suspension. In the spectrum taken over many shots, the periodicity was smeared out due to random (uncorrelated) peak positions in different shots as shown in Fig 1. For suspensions with different particle concentration but same dye molarity, while the scattering mean free path $l_{s}$ was varied by more than one order of magnitude, the frequency spacing of lasing peaks barely changed. This means that the cavity length $L_{c}$ did not depend on $l_{s}$. Another characteristic length scale was the penetration depth of the pump light $L_{p}$, which determined the length of the pump cone. In the case of linear absorption, $L_{p}$ is on the order of the absorption length $l_{a}$. However, our pumping was so strong that it saturated the absorption of dye molecules. The saturation photon flux density $I_{s}=1 / \sigma_{f} \tau_{f}$, where $\sigma_{f}$ is the fluorescence cross section and $\tau_{f}$ is the lifetime of dye molecules in the excited state. For Rhodamine 640 molecules, $\sigma_{f}$ is of the order $10^{-16} \mathrm{~cm}^{2}$, and $\tau_{f} 10^{-9} \mathrm{~s}$. Thus, $I_{s} \sim 10^{25} \mathrm{~cm}^{-2} \mathrm{~s}^{-1}$. The typical pump pulse energy at the lasing threshold was $\sim 0.1 \mu \mathrm{J}$. From the pump pulse duration and pump spot size, we estimated that the incident pump photon flux density $I_{p} \sim 10^{27} \mathrm{~cm}^{-2} \mathrm{~s}^{-1}$, which was two orders of magnitude higher than $I_{s}$. Therefore, at the lasing threshold the absorption of dye molecules was strongly saturated, and the penetration depth $L_{p}$ was much longer than $l_{a}$. Assuming every dye molecule inside the pump cone absorbed one pump photon per pulse, we estimated the cone length $L_{p}=E_{p} /\left(h \nu_{p}\right) /\left(D \pi r_{p}^{2} / 3\right)$, where $E_{p}$ is the incident pump pulse energy, $h$ is the Planck constant, $\nu_{p}$ is the pump frequency, $D$ is the density of dye molecules, $r_{p}$ is the base diameter of the pump cone. The pump pulse was so short that the probability of a dye molecule being excited more than once during one pump pulse was negligible. For $E_{p}=0.1 \mu \mathrm{J}, \lambda_{p}=532 \mathrm{~nm}, D$ $=3.1 \times 10^{24} \mathrm{~m}^{-3}, r_{p}=20 \mu \mathrm{m}$, we got $L_{p}=236 \mu \mathrm{m}$. This value is close to the lasing cavity length estimated from the wavelength spacing of lasing modes, $L_{c}=265 \mu \mathrm{m}$. To confirm $L_{c} \sim L_{p}$, we changed $L_{p}$ by varying the dye concentration $D$. The higher the dye concentration, the shorter the penetration depth. If $L_{c} \sim L_{p}$, the spacing of lasing peaks $\Delta \lambda$ should increase. Figure 4(a) shows the single shot lasing spectra from three solutions of $M$ $=3,5,10 \mathrm{mM} / \mathrm{L}$. It is evident that the spacing of lasing peaks increased at higher dye concentration. Images of the pump cones in the inset of Fig. 4(b) directly show that the pump cone was longer in the solution of lower dye concentration. Figure 4(b) plots the spectral correlation functions for the three spectra in 4(a). From them we extracted $\Delta \lambda=0.34,0.48,0.96 \mathrm{~nm}$ for $M=3,5,10 \mathrm{mM} / \mathrm{L}$. This result confirmed that the lasing cavity length was determined by the pump penetration depth.

Since the lasing cavity length was approximately equal to the length of pump cone, the "mirrors" of the lasing cavity were located at the tip and base of the cone. The next question is what the mirrors were. The studies presented earlier ruled out the front windows of the cuvette, and the back window is too far away to affect the laser cavity since $L_{c}$ is much less than the cuvette width $(1 \mathrm{~cm})$. The mirrors could not be formed by the nonlinear change of refractive index (both its real and imaginary parts) of the dye solution under pumping, otherwise lasing should occur also in the neat dye solution without particles. Another possibility is that the particles aggregated in the solution to form large clusters that served as mirrors. We monitored the solution during the experiment by imaging it onto a CCD camera through a side window of the cuvette. No clusters of size larger than $1 \mu \mathrm{m}$ were observed in the pumped solution. To remove the clusters smaller than $1 \mu \mathrm{m}$, we passed the solution through a $0.45 \mu \mathrm{m}$ pore-size filter paper. After the filtering, lasing phenomena remained the same. We also tried other particles such as $\mathrm{ZnO}$ and $\mathrm{SiO}_{2}$ in different solvents like methanol and DEG. Our previous studies confirmed the particles did not aggregate in some of these resolutions where we observed lasing. Therefore, the mirrors were not clusters of particles. Another candidate was bubbles or shock waves that were generated by the pump pulse. ${ }^{9-11}$ We indeed observed bubbles in the solution when the pump beam was strong enough and its focal spot was close to the front window of cuvette. However, when the bubbles were large enough to be seen with our imaging apparatus, lasing peaks disappeared. Hence, large bubbles did not facilitate lasing. Small bubbles, which were too small to be invisible, might be generated when the pumping was not very high. Such bubbles were usually generated at the focal spot of the pump beam where the pump intensity was the highest. Thus the bubble formation should be sensitive to the distance between the focal spot and the front window that affected the pump intensity at the focal spot due to absorption in the solution. However, the lasing behavior remained the same when we shifted the focal spot by moving the lens. This result eliminated the possibility of small bubbles contributing to lasing. All the data we collected experimentally led us to think that the lasing cavity was formed by the particles themselves, in particular, by a pair of particles located near the tip and base of the pump cone. In the next section, we will present a simple theoretical mode for such a laser. 

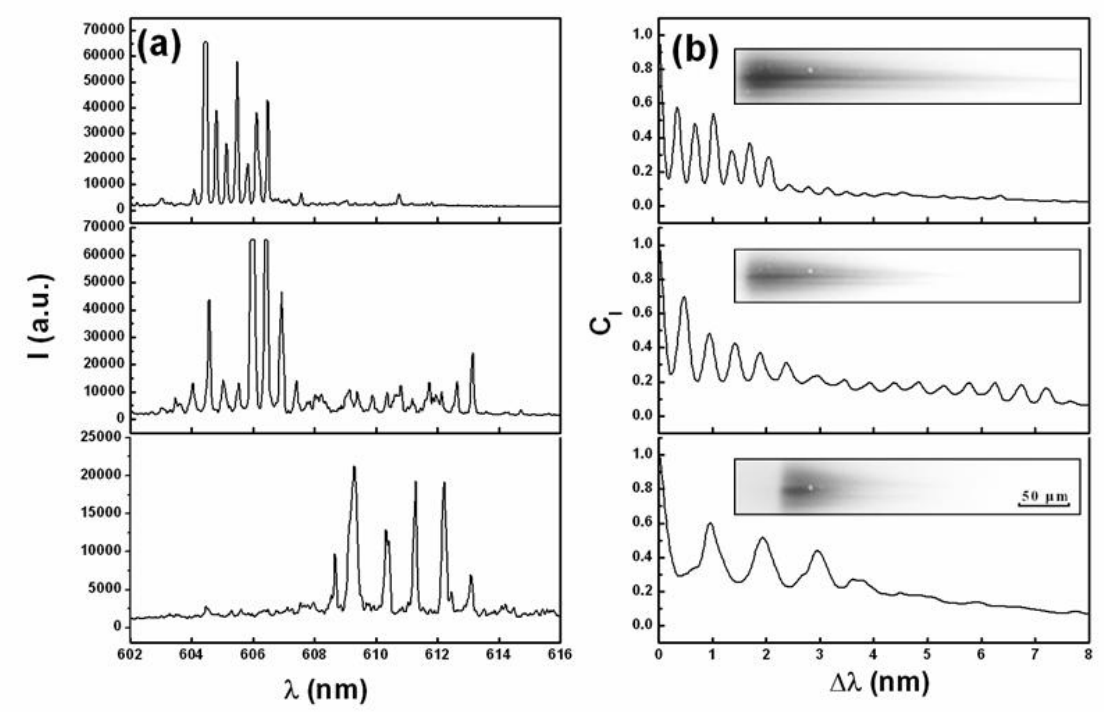

Figure 4. (a): Single shot emission spectrum from colloidal solution of $3.0 \times 10^{9} / \mathrm{cm}^{3} \mathrm{TiO}_{2}$ in DEG with different dye molarity. From top to bottom: $3 \mathrm{mM} / \mathrm{L}, 5 \mathrm{mM} / \mathrm{L}$, and $10 \mathrm{mM} / \mathrm{L}$. (b) Spectral correlation of corresponding lasing spectrum for these three dye molarity. Insets are side images of pumped region for each solution.

\section{THEORY}

Our interpretation of lasing in a dilute suspension of particles was based on a theory of Wilhelmi. ${ }^{6}$ In 1998, he studied analytically a laser composed of two Rayleigh scatterers in a homogeneous gain medium. Since our particles are Mie scatterers instead of Rayleigh scatterers, we generalized his model to laser resonator made of two Mie scatterers. In particular, we consider two spherical particles embedded in a homogeneous gain medium and separated by a distance $L_{c}$. In the Cartesian coordinates, the two scatterers, labeled A and B, are located at $(0,0,0)$ and $\left(0,0,-L_{c}\right)$. It is assumed that $L_{c}$ is much longer than the lasing wavelength $\lambda$, and the radius of the particles $r_{s}$ is less than $\lambda$. The diffraction loss in such an unstable resonator is very large. Thus one-path light amplification (from one scatterer to the other) must be very strong in order to compensate the loss. Since $L_{c} \gg \lambda>r_{s}$, the light propagating from one particle to the other can be approximated as a plane wave when it reaches the other particle. For example, the field incident onto particle A from B can be treated as a plane wave propagating along the $z$-axis. Note that the light originating from the spontaneous emission near particle A cannot be considered as a plane wave when it reaches particle A because of short propagation path. However, its intensity is negligibly small compared to that from particle B which experiences strong amplification owing to long propagation path. According to Mie's theory, the electric field scattered by particle A into the far field zone can be written as $^{12,13}$

$$
E_{j}=S_{j}(\theta, \varphi) \frac{e^{i k r}}{k r} E_{0 j}
$$

where $j=1$ or 2 refers to the field component perpendicular or parallel to the scattering plane formed by the incident wave vector $\mathbf{k}_{i n}$ and scattered wave vector $\mathbf{k}_{s c}, \theta$ and $\varphi$ denote the direction of $\mathbf{k}_{s c}, E_{0}$ represents the 
electric field amplitude of the incident plane wave, $S_{j}(\theta, \varphi)$ is the diagonal component of the amplitude scattering matrix $S$ for the Mie scatterer. For back-scattering $\theta=\pi, S_{1}$ and $S_{2}$ are independent of $\varphi$. Due to symmetry, $S_{1}(\theta=\pi)=S_{2}(\theta=\pi) \equiv S_{b}$. The polarization of the back-scattered field $E_{b}$ is equal to that of the incident field $E_{0}$. Therefore,

$$
E_{b}=S_{b} \frac{e^{i k r}}{k r} E_{0}
$$

In the presence of optical gain, $k$ becomes a complex number. Namely, $k=k_{r}+i g_{e} / 2$, where the net gain coefficient $g_{e} \equiv g-\alpha>0, g$ and $\alpha$ denote the gain and absorption coefficients respectively.

The field back-scattered by particle A is amplified as it propagates toward particle B. The field amplitude grows exponentially until it reaches particle B and is scattered again. Then the back-scattered wave travels back to particle A. To realize lasing, the electric field after one round trip shall be equal to the original field. The lasing threshold is

$$
\left(\frac{S_{b}}{k_{r} L_{c}}\right)^{2} e^{2 i k_{r} L_{c}+g_{e} L_{c}}=1 .
$$

The above equation sets the requirements for both phase and amplitude. The phase delay after one round trip should equal an integer multiple of $2 \pi$, i.e.

$$
2 k_{r} L_{c}+2 \phi_{b}=2 \pi m
$$

where $\phi_{b}$ is the phase of $S_{b}$, and $m$ is an integer. Eq.(4) selects the lasing frequency

$$
\nu_{m}=\frac{c}{2 \pi n_{e} L_{c}}\left(m \pi-\phi_{b}\right)
$$

where $n_{e}$ is the refractive index of the medium. The amplitude requirement in eq.(3) is

$$
\frac{\sigma_{b}}{L_{c}^{2}} e^{g_{e} L_{c}}=1
$$

where $\sigma_{b}=\left|S_{b}\right|^{2} / k_{r}^{2}$ is the back-scattering cross section. Eq.(6) determines the threshold gain coefficient

$$
g_{e}=\frac{2 \ln L_{c}-\ln \sigma_{b}}{L_{c}} .
$$

According to Eq.(5), the lasing modes are equally spaced in frequency. The wavelength spacing $\Delta \lambda \simeq$ $\lambda^{2} / 2 n_{e} L_{c}$. From the experimental values of $\Delta \lambda$ in Fig. 4 , we derived $L_{c}=372,265,134 \mu \mathrm{m}$ for three solutions of $M=3,5,10 \mathrm{mM} / \mathrm{L}$. According to Mie's theory, the back-scattering cross section $\sigma_{b}$ of a TiO $\mathrm{T}_{2}$ sphere with radius $200 \mathrm{~nm}$ in DEG at $\lambda=608 \mathrm{~nm}$ is about $0.05 \mu \mathrm{m}^{2}$. Using Eq.(6), we computed the threshold gain coefficient to be 400,534 and $955 \mathrm{~cm}^{-1}$, respectively. To check whether such high gain values can be reached in the dye solutions, we did a simple estimation of the maximum gain coefficient $g_{m}=\sigma_{f} N_{e x}$. $\sigma_{f}$ is the fluorescence cross section of rhodamine 640 molecule. Its value at the lasing wavelength $606-610 \mathrm{~nm}$ is about $5.0 \times 10^{-16}$ $\mathrm{cm}^{2} .{ }^{14} N_{e x}$ denotes the maximum density of excited dye molecules. For $M=3,5,10 \mathrm{mM} / \mathrm{L}, N_{e x}=1.8 \times 10^{18}$, $3 \times 10^{18}, 6 \times 10^{18} \mathrm{~cm}^{-3}$. Thus $g_{m}=900,1500$ and $3000 \mathrm{~cm}^{-1}$. In all three cases, the threshold gain coefficients are significantly smaller than $g_{m}$. Experimentally, we also measured the gain coefficients in neat dye solutions using the standard variable strip method. ${ }^{15}$ For example, in the $5 \mathrm{mM} / \mathrm{L}$ Rhodamine 640 solution, the effective gain coefficient $g_{e}$ was measured to be $26 \mathrm{~cm}^{-1}$ at the pump fluence $2.3 \times 10^{-4} \mathrm{~J} / \mathrm{cm}^{2}$. A linear extrapolation gives $g_{e}=900 \mathrm{~cm}^{-1}$ at the lasing threshold pump fluence $8.0 \times 10^{-3} \mathrm{~J} / \mathrm{cm}^{2}$. The extrapolated value is larger than the actual gain coefficient in the lasing experiment because the threshold pump fluence is given at the entrance of pump beam into the solution. As the pump beam propagates in the suspension, the pump fluence is reduced, so is the gain coefficient. Therefore, the threshold gain coefficient predicted by Eq.(6) is an average value.

Eq.(6) reveals two competing factors in determining the lasing threshold. On one hand, the optical feedback from one particle to the other is inversely proportional to $L_{c}^{2}$. The farther apart the two particles, the higher 
the loss of the two-scatterer cavity. On the other hand, light amplification grows exponentially with $L_{c}$. Owing to high optical gain in our experiment, the exponential growth of amplification dominates over the quadratic increase of cavity loss. The lasing threshold decreases with increasing $L_{c}$. Hence, the resonator with the lowest lasing threshold is composed of two particles with the largest separation inside the gain volume. In other words, the lasing cavity length is equal to the largest dimension of the gain volume. In a dilute suspension of particles, the gain volume is cone-shaped, thus the two particles farthest apart are located at the base and tip of the cone. The lasing cavity length is equal to the cone length. For two particles of separation $L_{c}$ shorter than the cone length, higher pumping is needed to reach the lasing threshold. If $L_{c}$ exceeds the cone length, one of the two particles is outside the gain volume. The larger value of $L_{c}$ does not lead to more amplification, because there is no stimulated emission outside the gain volume. However, the feedback gets weaker and the cavity becomes more lossy. As a result, the lasing threshold is higher. Hence, the lasing threshold is the lowest in the cavity of length $L_{c}$ approximately equals the pump cone length $L_{p}$.

The above analysis illustrates that the pair of scatterers that form the lasing cavity is selected by the maximum light amplification instead of the lowest diffraction loss. Unlike the usual case, the lasing cavity is not the one that has the lowest loss, but the one that extracts the largest gain. Experimentally, the gain volume contains many particles even in a dilute suspension of particles. For example, at $\rho=3.0 \times 10^{9} \mathrm{~cm}^{-3}$, the average particle spacing $d_{s}=\rho^{-1 / 3} \approx 7 \mu \mathrm{m}$, much shorter than the cavity length $L_{c}$. This has two consequences. First, it is highly probable to find two particles near both ends of the pump cone. Because $d_{s} \ll L_{c}$, the fluctuation of cavity length from $L_{c}-2 d_{s}$ to $L_{c}+2 d_{s}$ is much smaller than the average value $L_{c}$. It leads to small variation in lasing threshold and mode spacing from shot to shot, even though the particles move constantly in the solution. Secondly, the presence of a third particle in between the two that form the lasing cavity would cause additional scattering loss and increase the lasing threshold. The probability of such event rises with the particle density. In a simple estimation, we consider a cylinder of length $L_{c}$ and area $\sigma_{s} . \sigma_{s}$ is the total scattering cross of a single particle. When the number of particles inside this cylinder $N_{c}=\rho L_{c} \sigma_{s} \geq 2$, it is likely that light experiences an additional scattering as it propagates between the two particles separated by $L_{c}$. The corresponding particle density $\rho>\rho_{c}=1.5 \times 10^{10} \mathrm{~cm}^{-3}$ for $L_{c}=265 \mu \mathrm{m}$ at $M=5 \mathrm{mM} / \mathrm{L}$. This estimation agrees with the experimental observation that the lasing peaks start to decrease at $\rho=1.3 \times 10^{10} \mathrm{~cm}^{-3}$ (Fig. 1). Note that when $N_{c}>2$, the probability of forming two-particle cavity of length $L_{c}$ is low but not zero. Occasionally such a cavity is formed when particles move around in the solution, and lasing occurs at $\rho>\rho_{c}$. Increasing the particle density also changes the shape of gain volume from cone to hemisphere (Fig. 3). The maximum dimension of the gain volume is reduced, so is the length of two-particle cavity. According to Eq.(6), the lasing threshold rises. As the particle density keeps decreasing, the lasing threshold eventually rises as shown in left inset of Fig. 1. With an increase of particle spacing, the chance of find a pair of particles exactly at two ends of the pump cone is reduced. In other words, the fluctuation of the lasing cavity length is increased. No matter the two-scatterer cavity becomes shorter or longer than the pump cone, the lasing threshold always increases.

In addition to two-scatterer cavity, we also studied lasing in cavities formed by multiple scatterers. A ring cavity made of more than two particles in the cone-shaped gain volume has higher lasing threshold than the two-particle cavity of length $L_{p}$. As an example, let us consider a triangle loop formed by three particles. Their distances are $L_{1}, L_{2}$, and $L_{3}$. The lasing threshold condition can be derived in a similar way as that of a two-scatterer cavity:

$$
\frac{\sigma_{1}}{L_{1}^{2}} e^{g_{e} L_{1}} \frac{\sigma_{2}}{L_{2}^{2}} e^{g_{e} L_{2}} \frac{\sigma_{3}}{L_{3}^{2}} e^{g_{e} L_{3}}=1
$$

where $\sigma_{1}, \sigma_{2}$, and $\sigma_{3}$ denote the differential scattering cross-sections of the three particles. In the cone-shaped gain volume of length $L_{p}, L_{i} \leq L_{p}$, where $i=1,2,3$. Even $\sigma_{i}$ can be larger than $\sigma_{b}$ for some angle close to forward direction, it can be easily proved that the threshold for such triangle to lase is still higher than the threshold for two-scatterer cavity, namely $\prod_{i=1}^{3}\left(\sigma_{i} / L_{i}^{2}\right) e^{g_{e} L_{i}}<1$ where $g_{e}$ is the threshold gain for two-scatter cavity $L_{p}$. Similar conclusion can be drawn to ring cavities formed by more than three particles. Hence, the two-particle resonator of length $L_{p}$ has the lowest lasing threshold among all the closed loops inside the cone-shaped gain volume. Another type of multi-scatterer cavity is formed not by a single closed loop, but by many coupled loops. The coupling between two loops is caused by common scatterer(s) shared by both loops. The lasing threshold in 
this multi-particle cavity with distributed feedback can be lower than that of a two-scatterer cavity. The lasing modes in such a cavity are usually not equally spaced in frequency. Indeed experimentally we observed nonperiodic lasing peaks in some shots. Such phenomenon appeared more often at larger particle density where the chance of forming a multi-particle cavity with low loss is higher. Of course, the non-periodicity of spectral peaks could also be explained by simultaneous lasing in several two-particle cavities that are uncoupled. The chance of having more than one lasing cavities formed by independent pairs of particles is larger in solutions of higher dye concentration, because the aspect ratio of the pump cone is smaller. Further study is needed to determine which of the above two mechanisms is mainly responsible for the non-periodic lasing peaks. Nevertheless, the existence of periodic lasing peaks and the fact that their spectral spacing scales with the pump penetration depth at low particle density present clear evidences for lasing in two-scatterer cavities. The high optical gain is essential in selecting the lasing cavity, as the feedback from nearby particles is negligibly small compared to that from a particle far away. Strong amplification can modify the distributed feedback, making the lasing cavity differ from that of a passive system.

\section{CONCLUSION AND DISCUSSION}

In summary, we observed lasing in dye solutions containing a small amount of nano-particles under optical pumping. The focused pump beam created a cone-shaped gain volume. The cone length is determined by the absorption of dye molecules since optical scattering is very weak. The lasing output is directional, indicating lasing is confined to the direction parallel to the cone. The lasing modes are almost equally spaced in frequency, and their spacing is inversely proportional to the cone length. The lasing oscillation stops when there are too many particles in the solution, suggesting it is completely different from random lasing. Based on detailed experimental and theoretical analysis, we conclude that lasing occurs in a cavity formed by a pair of particles located at two ends of the cone-shaped gain volume. The feedback for lasing is provided by single-particle scattering. Owing to large diffraction loss, high optical gain is needed to reach the lasing threshold. One-path light amplification from one scatterer to the other results in an exponential growth of light intensity with the particle separation $L_{c}$, while the diffraction loss increases as $L_{c}^{2}$. Because of high gain, the exponential growth of amplified light dominates over the quadratic increase of cavity loss. Hence, the resonator with the lowest lasing threshold is composed of two particles with the largest separation inside the gain volume. Since the shape of gain volume is a cone with high aspect ratio, the lasing cavity is formed by two particles located at the tip and base of the cone. Therefore, the lasing cavity is chosen by the maximum light amplification instead of the lowest cavity loss. Although there are many particles inside the gain volume, the feedback from nearby particles is negligibly small compared to that from a particle far away which is strongly amplified in the long propagation path.

The two-scatterer laser operates in the ballistic regime where the system size is smaller than the transport mean free path. Note that the system size can be approximated by the maximum dimension of gain volume $d_{g}$. The emitted light that escapes the excitation volume has little chance of returning, because the absorption length at the emission wavelength is shorter than the transport mean free path. We would like to emphasize that the two-scatterer laser is fundamentally different from random laser. Although random lasing has been realized in the sub diffusive regime, ${ }^{16,17}$ the feedback mechanism is still based on multi-particle scattering. Thus the threshold of random laser decreases with increasing particle density $\rho$. In contrast, the two-scatterer laser is destroyed at large $\rho$. In fact, a transition from two-particle lasing to random lasing can be realized by increasing the particle density. At large $\rho$, strong scattering of pump light changes the shape of gain volume from a cone to a hemisphere. A reduction in the aspect ratio of the gain volume increases the number of particle pairs with separation equal to $d_{g}$. Moreover, the distance from one particle to several particles may be equal to $d_{g}$, and the feedback from all these particles is equally strong. The lasing cavity is thus a multi-particle cavity with distributed feedback. Therefore, we can control the lasing process in a colloidal solution of dye and particles by varying the particle density and pump geometry, as the shape of excitation volume is determined by the pump beam radius, the absorption length, and/or transport mean free path. ${ }^{18-20}$ 


\section{ACKNOWLEDGMENTS}

The authors acknowledge Dr. Christian Vanneste for stimulating discussion. This work was supported by the

National Science Foundation under the grant no. ECS-0244457.

\section{REFERENCES}

1. H. Cao, Lasing in random media, Waves in Random Media 13, R1 (2003), and reference therein.

2. R. V. Ambartsumyan, N. G. Basov, P. G. Kryukov, and V. S. Letokhov, Light pulse propagation in a nonlinear amplifying medium, IEEE J. Quant. Electron. QE-2 442 (1966).

3. V. S. Letokhov, Generation of light by a scattering medium with negative resonance absorption, Sov. Phys. JETP 26, 835 (1968).

4. H. Cao, J. Y. Xu, S.-H. Chang, S. T. Ho, E. W. Seelig, X. Liu, and R. P. H. Chang, Spatial confinement of laser light in active random media, Phys. Rew. Lett. 84, 5584 (2000).

5. H. Cao, J. Y. Xu, E. W. Seelig, and R. P. H. Chang, Microlaser made of disordered media, Appl. Phys. Lett. 762997 (2000).

6. B. Wilhelmi, Laser action in unstable microresonators, Microwave and Optical Tech. Lett. 17, 111 (1998).

7. A. Yamilov, X. Wu, H. Cao, and A. L. Burin, Absorption-induced confinement of lasing modes in diffusive random media, Opt. Lett. 30, 2430 (2005).

8. Y. Ling, H. Cao, A. L. Burin, M. A. Ratner, C. Liu, R. P. H. Chang, Investigation of random lasers with resonant feedback, Phys. Rev. A 64, 063808 (2001).

9. A. A. Chastov and O. L. Lebedev, Nonlinear scattering of intense light by colloidal suspensions, Sov. Phys. JETP 31455 (1970).

10. V. I. Bezrodnyi, V. I. Vashchuk, and E. A. Tikhonov, Unstable spreading of intensive radiation in liquid, Sov. Phys. Tech. Phys. 2389 (1978).

11. A. Vogel, K. Nahen, D. Theisen, and J. Noack, Plasma formation in water by picosecond and nanosecond Nd:YAG laser pulses, IEEE J. Sel. Topic. Quant. Electron. 2847 (1996).

12. H. C. van de Hulst Light Scattering by Small Particles (Dover, New York, 1981).

13. C. F. Bohren, and D. R. Huffman, Absorption and Scattering of Light by Small Particles (John Wiley \& Sons, New York, 1983).

14. N. M. Borges, H. L. Fragnito, and A. Kiel, Excited-state spectroscopy of organic dyes by use of population gratings, J. Opt. Soc. Am. B 11, 2321 (1994).

15. K. L. Shaklee, and R. F. Leheny, Direct determination of optical gain in semiconductor crystals, Appl. Phys. Lett. 18, 475 (1971).

16. B. R. Prasad, H. Ramachandran, A. K. Sood, C. K. Subramanian, and N. Kumar, Lasing in active, submean-free path-sized systems with dense, random, weak scatterers, Appl. Opt. 36, 7718 (1997).

17. G. D. Dice, S. Mujumdar, and A. Y. Elezzabi, Plasmonically enhanced diffusive and subdiffusive metal nanoparticle-dye random laser, Appl. Phys. Lett. 86, 131105 (2005).

18. A. Z. Genack, and J. M. Drake, Scattering for super-radiation, Nature (London) 368, 400 (1994).

19. D. S. Wiersma, M. P. van Albada, and A. Lagendijk, Random laser, Nature (London) 373, 203 (1995).

20. A. A. Burkov, and A. Yu. Zyuzin, Correlation function of speckle in reflection from photonic paint, JETP Lett. 63, 878 (1996). 